\title{
Série temporal do suicídio no Brasil: o que mudou após o Setembro Amarelo?
}

\author{
Temporal suicide series in Brazil: what changed after the "Setembro Amarelo"?
}

Series de tiempo del suicidio en Brasil: ¿que cambió después del "Setembro Amarelo"?

Milena Edite Casé de Oliveira ${ }^{1 *}$, Kedma Anne de Lima Gomes², Waleska Fernanda Souto Nóbrega², Elaine Custódio Rodrigues Gusmão³, Rayane Dantas dos Santos², Ramonyele Gomes Franklin².

\section{RESUMO}

Objetivo: Analisar a prevalência das notificações de suicídio no Brasil antes e após o lançamento da Campanha Setembro Amarelo (CSA) pelo Ministério da Saúde. Métodos: Foram utilizados dados secundários contidos no Sistema de Informações sobre Mortalidade (SIM) e no Sistema de Informação de Agravos de Notificação (SINAN) disponibilizados pelo DATASUS. Resultados: O número de casos notificados por intoxicação exógena somou um total de 452.579 pessoas no Brasil, destas, 15\% (67.966) correspondiam à categoria "tentativa de suicídio" e 10\% (45.901) a causas indeterminadas. Quanto à classificação de transtornos mentais e de comportamento da CID-10, entre 2013 e 2016, os óbitos notificados por "transtorno depressivo recorrente" (F33) vitimaram um total de 236 pessoas, das quais $57 \%$ (134) eram mulheres. Conclusão: Mais da metade das mortes violentas no mundo tem como causa o suicídio, tornandoo um problema da saúde pública. No ano de 2015 foi iniciada a Campanha Setembro Amarelo (CSA), que atua na prevenção ao suicídio. As taxas de incidência apresentaram dados ascendentes após a implantação da CSA, permitindo o questionamento a respeito da efetividade do programa. Faz-se necessário acompanhar a campanha por um maior intervalo de tempo, a fim de compreender seus impactos futuros.

Palavras-chave: Suicídio, Política pública, Prevenção primária.

\begin{abstract}
Objective: To analyze the prevalence of suicide reports in Brazil before and after the launch of the "Campanha Setembro Amarelo" (CSA) by the Brazilian Ministry of Health. Methods: Secondary data contained in Mortality Information System (SIM) and Notifiable Diseases Information System (SINAN) provided by DATASUS. Results: The number of cases reported for exogenous intoxication comprised 452,579 people in Brazil, $15 \%$ $(67,966)$ of those corresponding to the "suicide attempt" category and $10 \%(45,901)$ to undetermined causes. Between 2013 and 2016, for the ICD-10 classification of mental and behavioral disorders, deaths reported by "recurrent depressive disorder" (F33) victimized a total of 236 people; 57\% (134) were women. Conclusion: Suicide comprises more than half of all violent deaths in the world, making it a public health problem. Although the CSA was launched in 2015 to prevent suicide, the increasing incidence rate after the implementation of the program brought up questions about its efficiency. It is necessary to monitor the campaign for a longer period to understand its future impacts.
\end{abstract}

Keywords: Suicide, Public policy, Primary prevention.

\footnotetext{
1Universidade Federal da Paraíba. João Pessoa - PB. *E-mail: milacdo1@gmail.com

2 Universidade Estadual da Paraíba, Campina Grande - PB.

${ }^{3}$ Universidade Federal de Campina Grande, Campina Grande - PB.
} 


\section{RESUMEN}

Objetivo: Analizar la prevalencia de informes de suicidio en Brasil antes y después del lanzamiento de la "Campanha Setembro Amarelo" (CSA) por parte del Ministerio de Salud. Métodos: Se utilizaron datos secundarios contenidos en Sistema de Información de Mortalidade (SIM) y Sistema de Información de Enfermedades de Notificación (SINAN) proporcionados por DATASUS. Resultados: El número de casos reportados por intoxicación exógena totalizó 452,579 personas en Brasil, de los cuales el 15\% $(67,966)$ correspondió a la categoría "intento de suicidio" y el $10 \%(45,901)$ a causas indeterminadas. En cuanto a la clasificación ICD-10 de los trastornos mentales y del comportamiento, entre 2013 y 2016, las muertes reportadas por "trastorno depresivo recurrente" (F33) victimizaron a un total de 236 personas, de las cuales el 57\% (134) eran mujeres. Conclusión: más de la mitad de las muertes violentas en el mundo son causadas por suicidio, lo que lo convierte en un problema de salud pública. En 2015, se lanzó la "Campanha Setembro Amarelo" (CSA), que trabaja para prevenir el suicidio. Las tasas de incidencia mostraron datos ascendentes después de la implementación de la CSA, lo que permitió preguntas sobre la efectividad del programa. Es necesario monitorear la campaña por un período de tiempo más largo, para comprender sus impactos futuros.

Palavras-clave: Suicidio, Política pública, Prevención primária.

\section{INTRODUÇÃO}

O suicídio é caracterizado como um fenômeno complexo e multicausal proveniente da interação entre fatores de ordem filosófica, antropológica, psicológica, biológica e social, que não dispõe de uma explicação universal. Tal ato, provocado pelo indivíduo com intenção de pôr fim à vida, apresenta-se como um grave problema de saúde pública, com impactos nas esferas: interpessoal, familiar, econômica e social (RIBEIRO JF, et al., 2016; LIMA FN e GONDIM DSM, 2019; FERRACIOLI NGM, et al., 2019)

De acordo com a Organização Mundial de Saúde (OMS), mais da metade das mortes violentas no mundo tem como causa o suicídio, ocupando o segundo lugar como causa de morte no mundo entre pessoas de 15 a 29 anos (WORLD HEALTH ORGANIZATION, 2014).

Os dados sobre suicídio são compilados por país/continente e em seguida divulgados pela Organização Mundial de Saúde. Quanto aos dados calculados para o ano de 2015 e divulgados em 2018, pode-se observar que a média global da taxa de suicídios por 100 mil foi de 10,7. Ao se observar as grandes regiões, verificase forte variação, desde o Mediterrâneo Leste $(3,8)$, passando por África $(8,8)$, as Américas $(9,6)$ e Sudeste da Ásia $(12,9)$ até a maior prevalência observada na Europa $(14,1)$. No que diz respeito à taxa brasileira, esta é de 6,3, situando-se bem abaixo da média global e das grandes regiões (OMS, 2018).

A ação suicida geralmente relaciona-se a problemas de saúde mental, podendo existir, também, ligação com doenças físicas crônicas ou doenças terminais (JULIÃO M e BARBOSA A, 2011). Shikida CD, et al. (2007) reforçam e apresentam fatores que podem estar relacionados ao ato do suicídio, a saber: saúde mental (desordens psíquicas e problemas relacionados ao abuso de drogas lícitas e ilícitas); histórico de suicídio na família; problemas pessoais (separação/divórcio, perda da saúde ou da identidade); eventos estressantes (imposição, violência sexual e/ou corporal, instabilidade na família, alterações sociais e outros); acesso a dispositivos fatais (armas de fogo); problemas relacionados à lei (prisão), além de questões relacionadas à identidade sexual.

Para cada suicídio, são atribuídas de 10 a 25 tentativas que não deram certo, isto é, metade das pessoas que se mataram já haviam tentado consumar o ato anteriormente e, isto posto, $\mathrm{o}$ ato da tentativa de suicídio torna-se um importante fator de risco para o suicídio (BOTEGA NJ, 2014).

Por conseguinte, o suicídio implica consequências materiais e psíquicas, que envolvem a perda de um ser humano, produzindo reações negativas de ordem psicológicas para famílias, amigos e pessoas que fazem parte da rede de relações do envolvido e, considerando também o plano macrossocial, o suicídio favorece perdas socioeconômicas, pois a maioria das vítimas fazem parte de uma população economicamente ativa 
(MACHADO DB e SANTOS DN, 2015). Outrossim, os autores ainda mencionam que os impactos diante dos serviços de saúde merecem destaque, posto que envolvem despesas com atendimento em ambulatórios, cuidados de urgência e acolhimento de sequelas daqueles que sobrevivem (MACHADO DB e SANTOS DN, 2015).

O suicídio desperta a atenção da sua rede de apoio social mais próxima, uma vez que, constantemente, o indivíduo com ideação suicida não procura ajuda nos serviços de saúde mental, mas tem a tendência de desabafar com seus parentes e amigos, ou com profissionais de saúde que, por exemplo, trabalham na Atenção Primária (AP) (BRASIL, 2016).

As pessoas atendidas em serviços de saúde em consequência à tentativa de suicídio, dependendo da gravidade do ato, frequentemente são direcionadas para o pronto-atendimento, emergência ou centros de terapia intensiva não podendo alcançar opção de tratamento em saúde mental (BRASIL, 2016).

No sentido de minimizar 10\% dos óbitos por suicídio até o ano de 2020 o Ministério da Saúde lançou o Plano de Ação em Saúde Mental (PASM-2013), no qual o Brasil é um dos países signatários (OPAS, 2018). Além da intenção de minimizar os óbitos, a Organização Pan-Americana de Saúde (OPAS) lançou o referido Plano com a finalidade de se ter um acompanhamento anual do número de óbitos e o controle do desenvolvimento de programas que visem à prevenção ao suicídio (OPAS, 2018).

Ademais, a OMS criou a Suicide Prevention Program (SUPRE), que se trata de um manual de prevenção do suicídio, o qual recomenda ações e políticas de assistência e prevenção aos Estados-Membros (WHO, 2012).

As estratégias de prevenção citadas anteriormente contemplam condições apropriadas e efetivas para o atendimento e tratamento dos indivíduos acometidos por sofrimento psíquico, assim como visa o domínio ecossistêmico dos fatores de risco. Para tanto, os elementos listados como fundamentais para as ações de prevenção ao suicídio relacionam-se à maior sensibilidade para identificar a presença de risco e a divulgação de informações apropriadas (BRASIL, 2016).

Ainda de acordo com o Ministério da Saúde (2017), um dos empecilhos na busca por ajuda que possa impedir a efetivação do ato, é o preconceito relacionado à temática do suicídio. Nesse sentido, uma intervenção profissional com uma atenção direcionada e especializada pode proteger pessoas em condições de risco e adversidade do ato suicida (COLLINS KRL, et al., 2018).

Uma das tentativas de intervenção focada em indivíduos com ideações suicidas foi iniciada em Brasília no ano de 2015 pelo Centro de Valorização da Vida (CVV), pelo Conselho Federal de Medicina (CFM) e pela Associação Brasileira de Psiquiatria (ABP), a Campanha "Setembro Amarelo", que atua na prevenção ao suicídio. Essa causa foi estimulada internacionalmente e divulgada pela Associação Internacional de Prevenção do Suicídio (IASP), considerando oficialmente o dia 10 de setembro o Dia Mundial de Prevenção ao Suicídio, no entanto, a campanha ocorre durante o ano inteiro.

A Campanha Setembro Amarelo foi criada com o objetivo de levar ao conhecimento da população as formas de prevenção ao suicídio e alertá-la a respeito desse problema não só no Brasil, mas no mundo. Para que tal movimento informativo ocorra é possível se utilizar das informações gráficas e numéricas das taxas de suicídios ocorridos no país e como proceder em busca de orientação profissional caso necessária ajuda. Todas essas informações são disponibilizadas pelo Centro de Valorização da Vida (BEZERRA JJ e SILVA FV, 2019).

Diante do exposto e considerando que a Campanha Setembro Amarelo (CSA) tem como princípio as diretrizes de prevenção ao suicídio. Nesse sentido, o presente artigo tem como objetivo descrever a prevalência das notificações de suicídio no Brasil antes e após o lançamento da CSA pelo Ministério da Saúde.

\section{MÉTODOS}

Tratou-se de um estudo descritivo e ecológico de séries temporais. Os dados foram analisados através do software estatístico SPSS (versão 20.0). Foram utilizados dados secundários contidos no Sistema de 
Informações sobre Mortalidade (SIM) e no Sistema de Informação de Agravos de Notificação (SINAN), instituído pelo Ministério da Saúde do Brasil e disponibilizados pelo endereço eletrônico do DATASUS. O mesmo funciona através do registro sistemático de dados referentes a Estatísticas Vitais, como taxas de mortalidade e sobrevivência, assim como, informações epidemiológicas e de morbidade.

Para localizar os arquivos, a sequência de acesso no SIM/DATASUS foi obtida através da seguinte sequência de comandos: Estatística vitais, Mortalidade (1996-2016), pelo CID-10 e Mortalidade Geral. A classificação escolhida pelo CID-10 foi: o transtorno depressivo recorrente (F33), considerando como causas da mortalidade (transtornos mentais e comportamentais (F59); envenenamento, intoxicação ou exposição à substância nociva (F108); lesões autoprovocadas voluntariamente (F109) e eventos cuja intenção é indeterminada). No SINAN/DATASUS, os comandos selecionados foram: Doenças e agravos de notificação (2007 em diante) e Intoxicação Exógena.

Devido ao fato de o DATASUS não possuir um link único de estratégia de busca de mortalidade por suicídio, foram escolhidos dois caminhos de análise, com a finalidade de obtenção do maior número de dados acerca do evento investigado.

O primeiro caminho buscou coletar dados sobre mortalidade por suicídio, e a opção selecionada foi o transtorno depressivo recorrente por a literatura apontar como um fator de risco para o suicídio (SHIKIDA CD, et al., 2007).

O segundo caminho foi através do SINAN, onde obteve-se dados acerca do número de tentativas de suicídio, também apresentado na literatura como fator de risco para a variável de interesse (BOTEGA NJ, 2014).

Variáveis como idade, sexo, escolaridade, mês e região de ocorrência também foram extraídas do sistema para o estudo. Diagnósticos pertencentes ao grupo CID-10 F33 (transtorno depressivo recorrente) também foram utilizados, com a finalidade de compreender a ocorrência de óbitos por região/unidade de federação no Brasil.

Como critérios de inclusão da amostra, foram integradas notificações registradas de tentativa de suicídio por Intoxicação Exógena, que englobam os seguintes aspectos: ignoradas/branco - "resposta ignorada ou não-respondida"; uso habitual; acidental; ambiental; uso terapêutico; prescrição médica; erro de administração; automedicação; abuso; ingestão de alimento; tentativa de suicídio; tentativa de aborto; violência/homicídio; outra. Ao final, optou-se pelo período compreendido entre os anos de 2013 e 2016 para "transtorno depressivo recorrente" e 2013 até 2017 para tentativas de suicídio, classificadas em "notificações por intoxicação exógena".

Os últimos períodos de ambas as buscas foram selecionados de acordo com a última atualização do banco de dados. A escolha de iniciar a análise a partir de 2013, justifica-se pela intenção de verificar as taxas de incidência e mortalidade relacionadas ao suicídio dois anos antes e dois anos após a criação da campanha "Setembro Amarelo", a fim de constatar possíveis mudanças nos referidos indicadores.

\section{RESULTADOS}

Os resultados da pesquisa mostram que, no Brasil, as taxas de incidência relacionadas ao suicídio apresentaram dados ascendentes após a implantação do programa, apontando uma variação de mortalidade de 45 para 75 em 2013 e 2016, respectivamente, correspondendo a um aumento de 66,6\% na taxa de incidência entre os anos supracitados, conforme notificações realizadas no DATASUS (Gráfico 1).

Esses resultados levantam questionamentos se esse aumento é devido a um efeito adverso da proposta pela campanha, ou devido a uma maior efetividade na notificação dos episódios suicidas.

Em todos os anos pesquisados, a maioria dos casos de óbito por "transtorno depressivo recorrente" se concentrou nas faixas etárias de 70 a 79 anos e 80 anos ou mais, representando, juntos, $36 \%$ das notificações. Os locais que apresentaram maior ocorrência foram o estado do Paraná com 17\% (40) da amostra, seguido por São Paulo, com 14\% (33). 
Gráfico 1 - Número de notificações de mortalidade por transtorno depressivo recorrente entre os anos de 2013-2016. Brasil, 2013-2016.
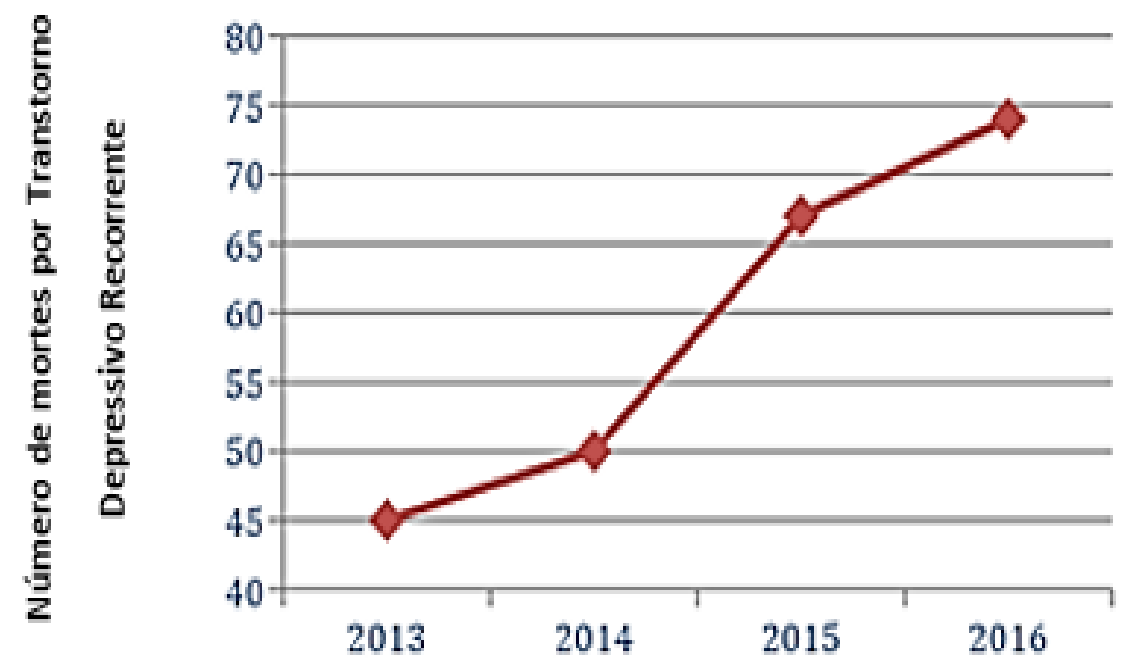

\section{Notificações}

Tempo

Fonte: MS/SVS/CGIAE - Sistema de Informações sobre Mortalidade - SIM (2013-2016).

Os dados mostraram que no ano de 2013 , os meses que mais registraram notificações por tentativa de suicídio foram setembro e outubro. Em relação aos anos de 2015 a 2017, os meses com mais notificações se concentraram no segundo semestre, especialmente entre os meses de setembro a novembro em 2015, de agosto a dezembro em 2016, e de agosto a outubro em 2017, com maior prevalência para o sexo feminino (54\%). Os achados são semelhantes aos dados do transtorno depressivo recorrente (Tabela 1).

Considerando a classificação de transtornos mentais e de comportamento da CID-10, entre 2013 e 2016 , os óbitos notificados por "transtorno depressivo recorrente (F33)" vitimaram um total de 236 pessoas, das quais $57 \%$ (134) eram mulheres e $43 \%$ (120) eram homens. O número de casos notificados por intoxicação exógena somou um total de 452.579 pessoas no Brasil, destas, 15\% (67.966) correspondiam à categoria "tentativa de suicídio" e 10\% (45.901) a causas indeterminadas (Tabela 1).

A Unidade Federativa que mais apresentou notificações para intoxicação exógena foi São Paulo, representando $25 \%$ dos casos notificados. Segundo o DATASUS, o número dos casos de óbito por intoxicação exógena no período 2013 e 2017 foi de 5.535, sendo a faixa etária mais prevalente a de 20 a 39 anos, representando $41 \%(2.277)$ da amostra. Em relação à etnia, a mais prevalente foi a branca, representando $42 \%$ (2.321) da amostra, seguida pela parda, com 36\% (2.007) do total que chegou a óbito por este fator. 


\section{Revista Eletrônica Acervo Saúde / Electronic Journal Collection Health ｜ ISSN 2178-2091}

Tabela 1 - Número de tentativas de suicídio, classificados em notificações por intoxicação exógena entre 2013-2017. Brasil, $2013-2017$.

\begin{tabular}{|c|c|c|c|c|c|c|c|c|c|c|c|c|c|c|c|}
\hline & & 2013 & & & 2014 & & & 2015 & & & 2016 & & & 2017 & \\
\hline Mês & $\mathbf{T}$ & IG/B & 0 & $\mathbf{T}$ & N.N & 0 & $\mathbf{T}$ & N.N & 0 & $\mathbf{T}$ & N.N & 0 & $\mathbf{T}$ & N.N & 0 \\
\hline Jan & 2603 & 895 & 5394 & 3036 & 1047 & 5354 & 3098 & 969 & 5300 & 2906 & 1037 & 5023 & 3753 & 1280 & 6136 \\
\hline Fev & 2596 & 945 & 4029 & 2950 & 1047 & 4779 & 2799 & 901 & 4509 & 2792 & 964 & 4602 & 3575 & 1119 & 5707 \\
\hline Mar & 2670 & 962 & 4186 & 3024 & 1075 & 5022 & 2897 & 1102 & 4784 & 2953 & 984 & 4186 & 3641 & 1215 & 5691 \\
\hline Abr & 2352 & 853 & 3679 & 2787 & 1024 & 4404 & 2563 & 874 & 3890 & 2714 & 854 & 3796 & 3688 & 1063 & 5123 \\
\hline Mai & 2342 & 868 & 3889 & 2497 & 924 & 4089 & 2463 & 753 & 3787 & 2639 & 901 & 3886 & 3782 & 1125 & 5148 \\
\hline Jun & 2338 & 770 & 3973 & 2605 & 897 & 4114 & 2358 & 772 & 3740 & 2549 & 925 & 3912 & 3378 & 1032 & 4782 \\
\hline Jul & 2341 & 855 & 4052 & 2448 & 901 & 4171 & 2520 & 773 & 4081 & 2841 & 966 & 4588 & 3495 & 1210 & 5158 \\
\hline Ago & 2685 & 998 & 4632 & 2831 & 1110 & 4602 & 2959 & 955 & 4324 & 3283 & 946 & 4782 & 4127 & 1151 & 5571 \\
\hline Set & 3050 & 1114 & 5133 & 2892 & 1014 & 4966 & 3174 & 1143 & 4808 & 3067 & 863 & 4376 & 4112 & 1141 & 5092 \\
\hline Out & 3116 & 1108 & 5240 & 3003 & 947 & 4771 & 3196 & 1047 & 5114 & 3632 & 972 & 4832 & 4443 & 1058 & 4712 \\
\hline Nov & 3013 & 1116 & 4906 & 2962 & 902 & 4621 & 3238 & 1017 & 4752 & 3658 & 1015 & 5088 & 2159 & 637 & 2209 \\
\hline Dez & 2951 & 998 & 5016 & 2698 & 931 & 4701 & 2875 & 878 & 4626 & 3282 & 989 & 5296 & 255 & 144 & 543 \\
\hline
\end{tabular}

Legenda: (T: Tentativas/ IG.B.: Ignorado ou em branco / O: Outros).

Fonte: Ministério da Saúde/SVS - Sistema de Informação de Agravos de Notificação - Sinan Net (2013 - 2017). 
Em relação ao nível de escolaridade, $62 \%$ (147) da amostra possuía até 7 anos de estudo, enquanto $38 \%$ (89) possuía 8 anos ou mais. No tocante à etnia, $50 \%$ (119) da amostra se autodeclarou branca, seguida da cor parda com 34\% (80) (Tabela 2).

Os meses que se destacaram em relação a maior ocorrência das notificações por transtorno depressivo recorrente foram Janeiro (10\%) e Outubro (10\%), seguido de Agosto (9\%) e Setembro (9\%). A população feminina se apresentou mais expressiva na amostra analisada (57\%).

Tabela 2 - Número de óbitos por ocorrência segundo sexo, CID-10 F33 (transtorno depressivo recorrente). Brasil, 2013-2016.

\begin{tabular}{cc}
\hline \multicolumn{2}{c}{ Número de óbitos por transtorno depressivo recorrente } \\
\hline Escolaridade & $\mathbf{N}(\%)$ \\
\hline$<$ anos de estudo & $147(62 \%)$ \\
$\geq 8$ anos de estudo & $89(38 \%)$ \\
\hline Etnia & $119(50 \%)$ \\
\hline Branca & $80(34 \%)$ \\
Parda & $37(16 \%)$ \\
\hline Ignorado ou em branco & $23(10 \%)$ \\
\hline Meses de maior ocorrência & $21(9 \%)$ \\
\hline Janeiro & $21(9 \%)$ \\
Agosto & $23(10 \%)$ \\
Setembro & $148(62 \%)$ \\
Outubro & \\
\hline Outros & $135(57 \%)$ \\
\hline Sexo & $101(43 \%)$ \\
\hline Feminino & \\
\hline Masculino &
\end{tabular}

Fonte: MS/SVS/CGIAE - Sistema de Informações sobre Mortalidade - SIM (2013 - 2016)

\section{DISCUSSÃO}

O presente estudo objetivou analisar a prevalência de notificação de suicídio no Brasil antes e após o a CSA. Os resultados apresentados demonstraram um aumento nas taxas de incidência nas notificações de suicídio após a implementação da CSA, levantando questionamentos acerca da eficácia do programa.

Tais achados corroboram com o estudo de Oliveira BM (2018), que evidenciou um aumento das taxas após o estabelecimento da CSA, atribuindo tal fato ao possível efeito contágio, onde acredita-se que a divulgação de notícias acerca do suicídio, com a disseminação de ideias e valores, tem a capacidade de reforçar ideações suicidas. Outro estudo realizado no Brasil, buscou analisar óbitos por suicídio entre as diferentes regiões do país, no período de 1996 a 2015. Os resultados apontaram um aumento progressivo nas taxas analisadas em todas as regiões no Brasil, sendo o Nordeste a região que apresentou a maior taxa de crescimento (JUNIOR AD, et al., 2019).

O estudo de Lisboa MR (2018) aponta que durante muito tempo, a divulgação de notícias sobre suicídio foi vetada por jornalistas nas redações de grandes empresas de comunicação no Brasil, e que esta realidade 
foi sendo alterada a partir do final do século passado, quando o silenciamento em torno desse problema de saúde pública foi gradativamente substituído por uma postura de exposição. Isso ocorreu apoiando-se na hipótese de que o silêncio sobre o tema não contribuiu para a proteção de quem, em algum momento, foi impelido a provocar a própria morte.

Assim, os serviços de saúde e a mídia vêm expandindo a divulgação sistemática de dados estatísticos sobre o suicídio, incluindo aqueles relativos a recortes populacionais, por região geográfica, faixa etária, sexo, nível educacional e motivações para o ato. No entanto, o autor traz a reflexão de que existem outros fatores intrínsecos ao fenômeno analisado, sendo, portanto, inviável se pensar na redução dos números de suicídios e tentativas de suicídio apenas pela via da racionalidade (LISBOA MR, 2018).

As duas variáveis utilizadas como estratégia de busca para compor a amostra foram os dados relativos à Transtorno Depressivo e Intoxicação Exógena. De acordo com a OPAS (2018) a depressão é um transtorno mental que acomete cerca de 300 milhões de pessoas em todo o mundo, podendo, em alguns casos, levar ao suicídio. A esse respeito, Tsegay A, et al. (2020), em um estudo caso-controle realizado na Etiópia, trouxeram o transtorno depressivo grave como fator significativo para a ideação suicida.

Santos AS, et al. (2013) consideram que a intoxicação exógena se apresenta entre os três principais meios utilizados nas tentativas de suicídio e nos casos de óbito por suicídio, estando $70 \%$ dos casos vinculados à substâncias como medicamentos e pesticidas. Em 2010, o Sistema Nacional de Informações TóxicoFarmacológicas (SINITOX) publicou que $17 \%$ dos registros de intoxicações estavam associados à indivíduos que tentaram suicídio, e $64,8 \%$ a óbitos relacionados ao suicídio. Dessa forma, acredita-se que as variáveis consideradas apresentaram uma boa explanação acerca do fenômeno estudado no Brasil.

Lamm M (2004) apresentou em sua pesquisa a mudança de indicadores de suicídio a partir de acontecimentos culturais, sociais e religiosos. O que pode justificar o fato dos meses de Fevereiro, Junho e Dezembro não terem apresentado os maiores números de tentativas no presente estudo, possivelmente por serem culturalmente meses festivos no Brasil, proporcionando diversão e afastamento temporário de questões que possam influenciar negativamente o emocional e incorrer em suicídio ou tentativa deste. Stack S (2000) ainda ressalta a ideia que a integração social presente nos feriados atua como um fator atenuante para o pensamento suicida.

A literatura aponta que o suicídio é predominante no sexo masculino, sendo, a taxa global de suicídio entre homens de 15 por 100 mil habitantes, e entre mulheres de 8 por 100 mil habitantes (WHO, 2014). Entretanto, ainda de acordo com a OMS (2014) as taxas de suicídio sofrem variação ao se considerar variáveis como região, país e idade. Nesse sentido, a razão entre a taxa de suicídio entre homens e mulheres nos países desenvolvidos é de 3,5 e nos países em desenvolvimento é 1,6 (WHO, 2014).

Uma justificativa plausível para achados contrários neste estudo é o fato de não haver a inclusão de outras situações notificadas como possíveis causas do suicídio, a exemplo de afogamentos, incêndios propositais e outras modalidades que poderiam incluir mais sujeitos do sexo masculino (VIANA GN, et al., 2008).

Por outro lado, Viana GN, et al. (2008) apresentam em sua pesquisa as mulheres como maioria nas tentativas de suicídio, corroborando com os achados deste estudo. Os dados referentes a escolaridade, cor de pele e regiões que apresentaram maiores notificações apresentaram consonância com a literatura (SOUZA VS, 2011; RIBEIRO JF, et al., 2018; MACHADO DB e SANTOS DN, 2015)

Entre idosos, Minayo MSC e Cavalcante FG (2010) defendem que os episódios suicidas podem relacionarse com as dificuldades em lidar com o processo de envelhecimento e suas consequências, como isolamento social, morte de um ente querido, doença terminal com dores incontroláveis, medo do prolongamento da vida sem dignidade, além das modificações nos papéis sociais que lhes conferiam reconhecimento e as situações de dependência física/mental diante das quais o idoso se sente humilhado.

Entre jovens, a motivação geralmente possui origem afetiva, como rompimento de relacionamentos, pressões acadêmicas e ocupacionais (frente à competitividade no mercado de trabalho), enquanto para os adultos e pessoas de meia idade, os aspectos relacionados com o trabalho e as cobranças do âmbito familiar 
são os fatores de risco mais expressivos (CARMO EA, 2018). Como fator comum para todas as idades, Abreu TO e Souza MB (2017) mostram que as tecnologias de informações do mundo contemporâneo, como computadores, acesso à internet e uso de celulares, revolucionaram a forma como as pessoas se comunicam, socializam, buscam e trocam informações, levando a um isolamento e provável ideação suicida.

\section{CONCLUSÃO}

O estudo apresentou um aumento das notificações de suicídio e de tentativa de suicídio após o início da Campanha Setembro Amarelo. A presente pesquisa pode servir como subsídio para novos estudos que busquem analisar a Campanha do Setembro Amarelo e suas reverberações na sociedade brasileira. Além da subnotificação inerente ao sistema, pode-se considerar o pouco tempo de implementação do CSA como uma limitação do presente estudo, sugerindo-se a investigação a longo prazo da efetividade desta política. Assim, torna-se necessária a sensibilização dos profissionais responsáveis pelo preenchimento dos dados e centralização das informações, uma vez que a falta destes, compromete a investigação e a formulação de campanhas para um público-alvo.

\section{REFERÊNCIAS}

1. ABREU TO, SOUZA MB. A influência da internet nos adolescentes com ações suicidas. 2017.

2. BEZERRA JJ, SILVA FV. As cores da vida: Estratégias biopolíticas nas campanhas setembro amarelo, outubro rosa e novembro azul. Revista Eletrônica do Netlli, 2019; 8(2):728-741.

3. BOTEGA NJ. Comportamento suicida: epidemiologia. Psicologia USP, 2014; 25(3): 231-236.

4. BRASIL. Ministério da Saúde. Suicídio. Saber, agir e prevenir. Boletim Epidemiológico, 2017; 48(30).

5. CARMO EA. Características sociodemográficas e série temporal da mortalidade por suicídio em idosos no estado da Bahia, 1996-2013. Epidemiol. Serv. Saúde, 2018; 27(1):e20171971.

6. CENTRO DE VALORIZAÇÃO DA VIDA (CVV). História do Setembro Amarelo. 2016.

7. COLLINS KRL, et al. Mind full of life: Does mindfulness confer resilience to suicide by increasing zest for life? Journal of Affective Disorders, 2018; 226, 100-107.

8. CONSELHO FEDERAL DE MEDICINA (CFM). CFM e ABP lançam campanha Setembro Amarelo para prevenção ao suicídio. Setembro, 2017.

9. FERRACIOLI NG, et al. Os bastidores psíquicos do suicídio: uma compreensão psicanalítica. Revista do NESME, 2019; 16(1): 1-17.

10. JULIÃO M, BARBOSAA. Depressão em Cuidados Paliativos. Acta Médica Portuguesa, 2011; 24(S4): 807-818.

11. JUNIOR AD, et al. Mortalidade por suicídio na população brasileira, 1996-2015: qual é a tendência predominante? Cadernos Saúde Coletiva, 2019; 27(1): 20-24.

12. LAMM M. Bem-vindo ao judaísmo. 2nd ed. São Paulo: Sêfer, 2004.

13. LIMA FN e GONDIM DSM. Hereditariedade e repetição no ato suicida: uma abordagem psicanalítica. Interdisciplinary Scientific Journal, 2019; 6(5): 354.

14. LISBOA MR. Do silencio à implicação. Reciis, 2018; 12(3): 249-251.

15. MACHADO DB, SANTOS DN. Suicídio no Brasil, de 2000 a 2012. J Bras Psiquiatr, 2015; 64(1): 45-54.

16. MINAYO MS, CAVALCANTE FG. Suicídio entre pessoas idosas: revisão da literatura. Rev. Saúde Pública, 2010; 44(4): $750-757$.

17. OLIVEIRA BM. Suicídio, setembro amarelo e efeito contágio um estudo ecológico em Santa Catarina. Trabalho de Conclusão de Curso (Graduação em Medicina). Universidade do Sul de Santa Catarina, Santa Catarina, 2018; 32p.

18. OPAS. Organização Pan-Americana de Saúde. Folha informativa - Depressão, 2018.

19. RIBEIRO JF, et al. Perfil sociodemográfico da mortalidade por suicídio. Revista de Enfermagem da UFPE, 2018; 12(1): 44-50.

20. SANTOS AS, et al. Suicídios e tentativas de suicídios por intoxicação exógena no Rio de Janeiro: análise dos dados dos sistemas oficiais de informação em saúde, 2006-2008. Rev. Brasileira de Epidemiologia, 2013; 16: 376-87.

21. SHIKIDA CD, et al. Teoria econômica do suicídio: estudo empírico para o Brasil. Análise Econômica, 2007; 25(47): 123-147.

22. SINITOX. Sistema Nacional de Informações Tóxico-Farmacológicas. Casos Registrados de Intoxicação Humana por Agente Tóxico e Circunstância, Brasil, 2010.

23. SOUZA VS. Tentativas de suicídio e mortalidade por suicídio em um município no interior da Bahia. J. bras. psiquiatr, $2011 ; 60(4): 294-300$. 
24. STACK S. Suicide: a 15-year review of the sociological literature part I: cultural and economic factors. Suicide Life Threat Behav, 2000; 30(2): 145-162.

25. TSEGAY A, DAMTE A, KIROS A. Determinants of suicidal ideation among patients with mental disorders visiting psychiatry outpatient unit in Mekelle town, psychiatric clinics, Tigray, Northern Ethiopia: a case-control study. Ann Gen Psychiatry, 2020; 19: 20.

26. VIANA GN. Prevalência de suicídio no Sul do Brasil, 2001-2005. J. bras. Psiquiatr, 2008; 57(1): 38-43.

27. WORLD HEALTH ORGANIZATION. WHO. World Health Statistics. 2012.

28. WORLD HEALTH ORGANIZATION. WHO. World Health Statistics. 2014. 